\title{
Fire Behavior of 3D-Printed Polymeric Composites
}

Karthik Babu, Oisik Das, Vigneshwaran Shanmugam, Rhoda Afriye Mensah, Michael Försth, Gabriel Sas, Ágoston Restás, and Filippo Berto

\author{
Submitted: 2 December 2020 / Revised: 12 February 2021 / Accepted: 18 February 2021 / Published online: 30 March 2021
}

\begin{abstract}
3D printing or additive manufacturing (AM) is considered as a flexible manufacturing method with the potential for substantial innovations in fabricating geometrically complicated structured polymers, metals, and ceramics parts. Among them, polymeric composites show versatility for applications in various fields, such as constructions, microelectronics and biomedical. However, the poor resistance of these materials against fire must be considered due to their direct relation to human life conservation and safety. In this article, the recent advances in the fire behavior of 3D-printed polymeric composites are reviewed. The article describes the recently developed methods for improving the flame retardancy of 3D-printed polymeric composites. Consequently, the improvements in the fire behavior of 3D-printed polymeric materials through the change in formulation of the composites are discussed. The article is novel in the sense that it is one of the first studies to provide an overview regarding the flammability characteristics of 3D-printed polymeric materials, which will further incite research interests to render AM-based materials fire-resistant.
\end{abstract}

$\begin{array}{ll}\text { Keywords } & \begin{array}{l}\text { additive manufacturing, fire behavior, flame } \\ \text { retardants, polymeric composites }\end{array}\end{array}$

\section{Introduction}

Thermo-compression (including extrusion and injection molding) is a classical technique applied in the production of polymer parts. However, the utilization of thermo-compression to produce parts with complex geometry is challenging. Hence, there has been the need to adopt 3D printing methods to facilitate the production process. 3D printing (or additive manufacturing) has gained popularity owing to its highly flexible nature (Ref 1). 3D printing processes such as fused deposition modeling (FDM), stereolithography (SLA), and selective laser sintering (SLS) are the well-established techniques for plastic parts manufacturing (Ref 2). The basic

This invited article is part of a special topical focus in the Journal of Materials Engineering and Performance on Additive Manufacturing. The issue was organized by Dr. WilliamFrazier, Pilgrim Consulting, LLC; Mr. Rick Russell, NASA; Dr. Yan Lu, NIST; Dr. Brandon D. Ribic, America Makes; and Caroline Vail, NSWC Carderock.

Karthik Babu, Department of Mechanical Engineering, Centurion University of Technology and Management, R.Sitapur, Odisha 761211, India; Oisik Das, Michael Försth, and Gabriel Sas, Structural and Fire Engineering Division, Department of Civil, Environmental and Natural Resources Engineering, Luleå University of Technology, 97187 Luleå, Sweden; Vigneshwaran Shanmugam, Department of Mechanical Engineering, Saveetha School of Engineering, Saveetha Institute of Medical and Technical Sciences, Chennai 602 105, Tamil Nadu, India; Rhoda Afriye Mensah, School of Mechanical Engineering, Nanjing University of Science and Technology, Nanjing 210094, China; Ágoston Restás, Department of Fire Protection and Rescue Control, National University of Public Service, Budapest 1011, Hungary; Filippo Berto, Department of Mechanical Engineering, Norwegian University of Science and Technology, 7491 Trondheim, Norway. Contact e-mails: oisik.das@ltu.se, odas566@aucklanduni.ac.nz, and filippo.berto@ntnu.no. operating principle is different for each process and each one has its advantages and drawbacks. The FDM process operates on the basis of melting and extrusion of thermoplastic polymer filament, which provides relatively low resolution and accuracy at the lowest cost (Ref 3 ). In the case of SLA, the laser cures the photo-polymeric material, which provides fine details with high resolution and accuracy (Ref 4). On the other hand, SLS uses a laser source to fuse the polymer powder and form the required shape. SLS produces plastic parts with good mechanical properties resembling injection molded parts. Additionally, high productivity and low-cost production can be possible with the SLS technique (Ref 5).

Among the aforementioned 3D printing techniques, FDM is a prevalent manufacturing method because of its low-cost printing. However, the range of feedstock materials-filament that is commercially available is limited. Nevertheless, in some specific 3D printing processes, no filaments are necessary. Polymer pellets can be directly fed into the printing nozzle thus, allowing researchers to investigate many polymeric matrices with no commercial limitation (Ref 6). Although, FDM-made polymers parts are capable of possessing acceptable mechanical strength and performance (Ref 7,8), they generally have poor flame-retardant properties (Ref 9). The poor flammability reflects negatively on the overall performance of these 3Dprinted products. Figure 1 illustrates the flame retardancy mechanism of 3D-printed parts and the role of flame retardants (FRs) during polymer pyrolysis.

Researchers have attempted to enhance the flame retardancy of numerous polymers since the past decades and significant findings were reported (Ref 10, 11). For instance, halogenbased FRs have been extensively used in the past to enhance the flame resistance of various polymeric materials, however, they are currently prohibited due to their adverse effects like environmental pollution and toxicity (through the evolved gases) toward humans ( $\operatorname{Ref} 12,13)$. Therefore, it was essential to develop halogen-free flame retardants to enhance the fire safety of polymeric materials. In this view, an array of FRs were developed like phosphorus-based (Ref 14, 15), silicone-based (Ref 16), metal hydroxides (Ref 17, 18), nitrogen-based (Ref 19) or carbon-family (such as fullerene, carbon nano tubes and 

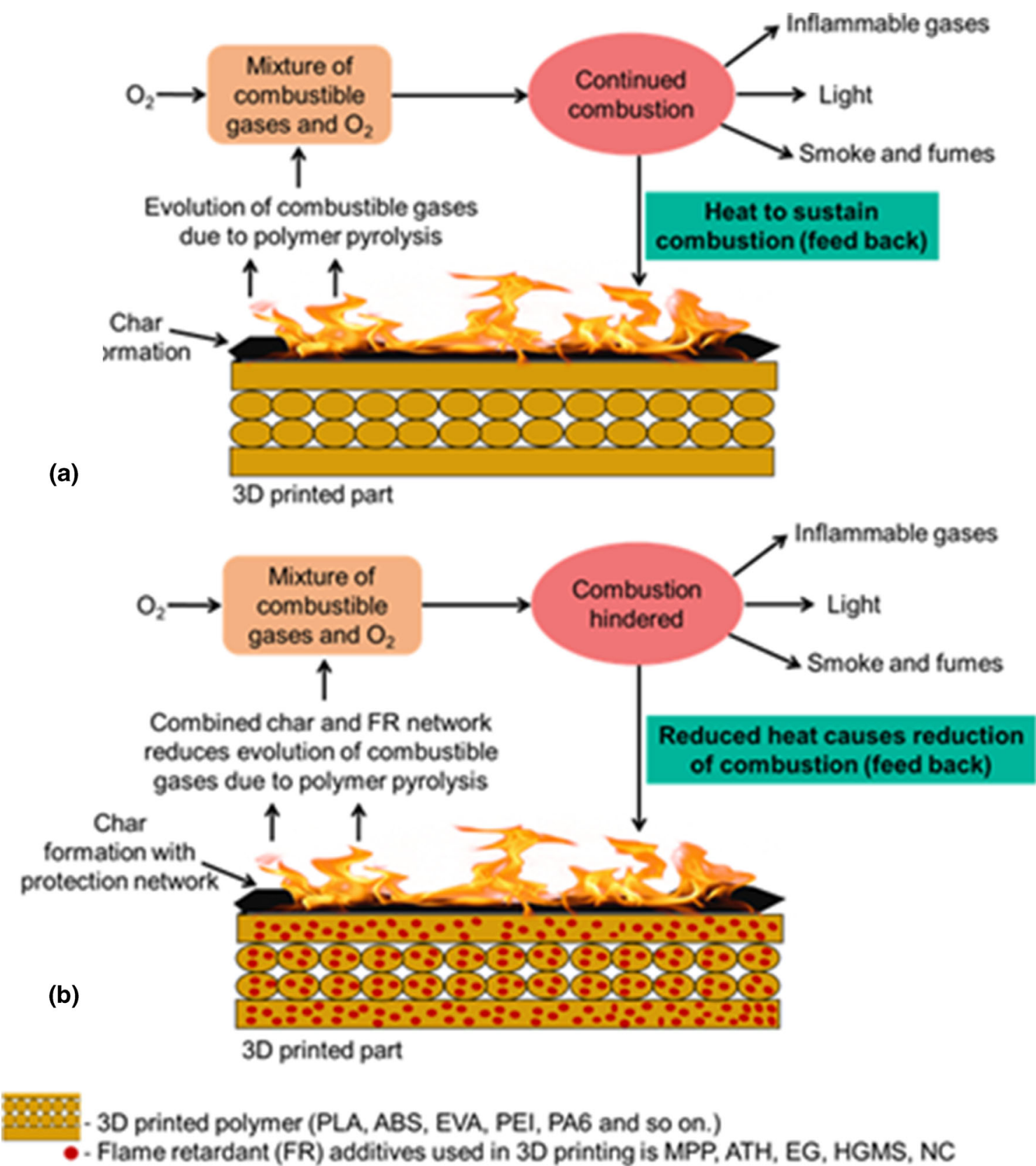

3D printed polymer (PLA, ABS, EVA, PEI, PA6 and so on.)

- Flame retardant (FR) additives used in 3D printing is MPP, ATH, EG, HGMS, NC and so on.

Fig. 1 Schematic diagram showing the behavior of a 3D-printed part without flame retardant (FR) (a) and with FR (b). PLA: Poly (lactic acid), ABS: Acrylonitrile butadiene styrene, EVA: Ethylene-vinyl acetate, PEI: Polyetherimide, PA6: Polyamide-6, MPP: Melamine polyphosphate, ATH: Aluminum trihydroxyde, EG: Expandable graphite, HGMS: Hollow glass microspheres, NC: Nanoclay

graphene) (Ref 20) to improve the flame retardancy of polymers. Among these, phosphorus-based flame retardants are frequently incorporated by chemical or physical methods to achieve superior flame retardancy of polymers (Ref 21, 22). For instance, $\mathrm{Xu}$ et al. (Ref 23) synthesized a phosphoruscontaining chemical agent to cure epoxy (as a reactive flame retardant), and revealed that the 5 wt.\% of phosphorus-based FR added epoxy successfully passed UL-94 test with V-0 flammability rating and the LOI value was as high as $30.8 \%$. Elsewhere, Huo et al. (Ref 24) introduced phosphorus element into the main chains of epoxy chemically and the $1 \mathrm{wt} \%$ phosphorus flame retardant added epoxy displayed a limiting oxygen index (LOI) of 38.3\% and passed in UL-94 burning test with a V-0 rating (Ref 24). Das et al. (Ref 25, 26) recommended biochar derived from pyrolysis of biomass as an effective filler to enhance the fire resistance characteristics of polymers. For instance, in their investigations, the peak heat release rate (PHRR) for biochar added PP composites was 318 and 281 $\mathrm{kW} / \mathrm{m}^{2}$, which was $73 \%$ and $70 \%$ lower than that of neat PP, however, the time to ignition (TTI) for biochar added PP composites was reduced to 13 and $16 \mathrm{sec}$, which was 45 to $55 \%$ lower than that for neat PP. Consequently, the total heat release (THR) was reduced by $9 \%$ and $6 \%$ compared to neat PP (Ref 25, 26).

It is worth noting that 3D-printed polymers and their composites play a vital role in electronic device fabrication (Ref 
27, 28), aerospace industry (Ref 29) and so on. Though beneficial for certain performance properties, these materials pose significant fire risks. Reinforcements used in polymers such as wood-based and other natural lignocellulosic materials are flammable but compared to many synthetic polymers these materials can have lower fire hazard (Ref 30-32). Fire is the major risk for most polymers and their composites since they are susceptible to combustion due to their hydrocarbon backbone. A well-designed plastic product must be safe, and for this purpose, the development of fire-proof materials is critical (Ref 33). The available manufacturing processes require 3D-printed polymers to have high strength, be thermally resistant and flame retardant. Among the aforementioned properties, mechanical and thermal properties such as tensile strength, flexural strength and thermal stability of 3D-printed polymeric materials are well documented (Ref 7, 34, 35). However, only a very few studies have investigated the fire behavior of 3D-printed polymer parts. This article, therefore, seeks to review the fire behavior of 3D-printed polymeric composites. The review article provides an insight into the various polymers and their composites used in $3 \mathrm{D}$ printing and the various flame-retardant treatments applicable in this field. Moreover, the contribution of the industrial sector toward the improvement of the fire retardancy of 3D printing materials is analyzed. Finally, recommendations are provided to garner further research toward comprehending the fire behavior of these 3D-printed polymeric materials.

\section{Flame Retardancy of 3D-Printed Polymer Parts}

In light of the above discussion, the flammability of the polymer and its composites need to be reduced to increase their use, especially in high risk applications. The flammability of polymers and their composites can be enhanced by the addition of suitable FRs. Therefore, polymeric materials' tendency to combust upon exposure to fire must be reduced by the incorporation of different FRs (Ref 36-43). Some of the available FR strategies for polymeric materials are presented in the schematic diagram shown in Figure 2. Polymer products made from 3D printing technique are also not exempted from the problem of vulnerability toward fire (Ref 44), which warrants several FR treatments. In the following sections, the fire behavior of various types of polymers and their composites processed by AM are discussed in detail.

\subsection{Poly (Lactic Acid) (PLA)}

FDM is one of the frequently employed 3D printing techniques for the processing of conventional thermoplastic polymers such as PLA, Acrylonitrile Butadiene Styrene (ABS), polyamide (PA), and Polyethylene Terephthalate (PET). Among these polymers, PLA is one of the widely used materials in the FDM process. By choosing the optimal PLA grade (molecular weight, the proportion of D- or L-enantiomers, crystalline morphology, etc.), it is conceivable to achieve PLA-based polymers with satisfying mechanical and thermal properties considering the explicit application (Ref 45).

PLA is a biodegradable material having excellent mechanical properties and optical transparency, which are very attractive features in several applications (Ref 46). However, PLA has high flammability that restricts their application in wide range of applications. Addition of flame retardants (in the form of nano-filler) in PLA is a viable strategy to overcome this shortcoming (Ref 47, 48). The use of nano-filler permits the simultaneous enhancement of material's properties and processability. Drawability and processability are the two influencing parameters in FDM technology because they have roles on both PLA feedstock filament production and layer deposition during the printing of PLA. In this regard, addition of FRs

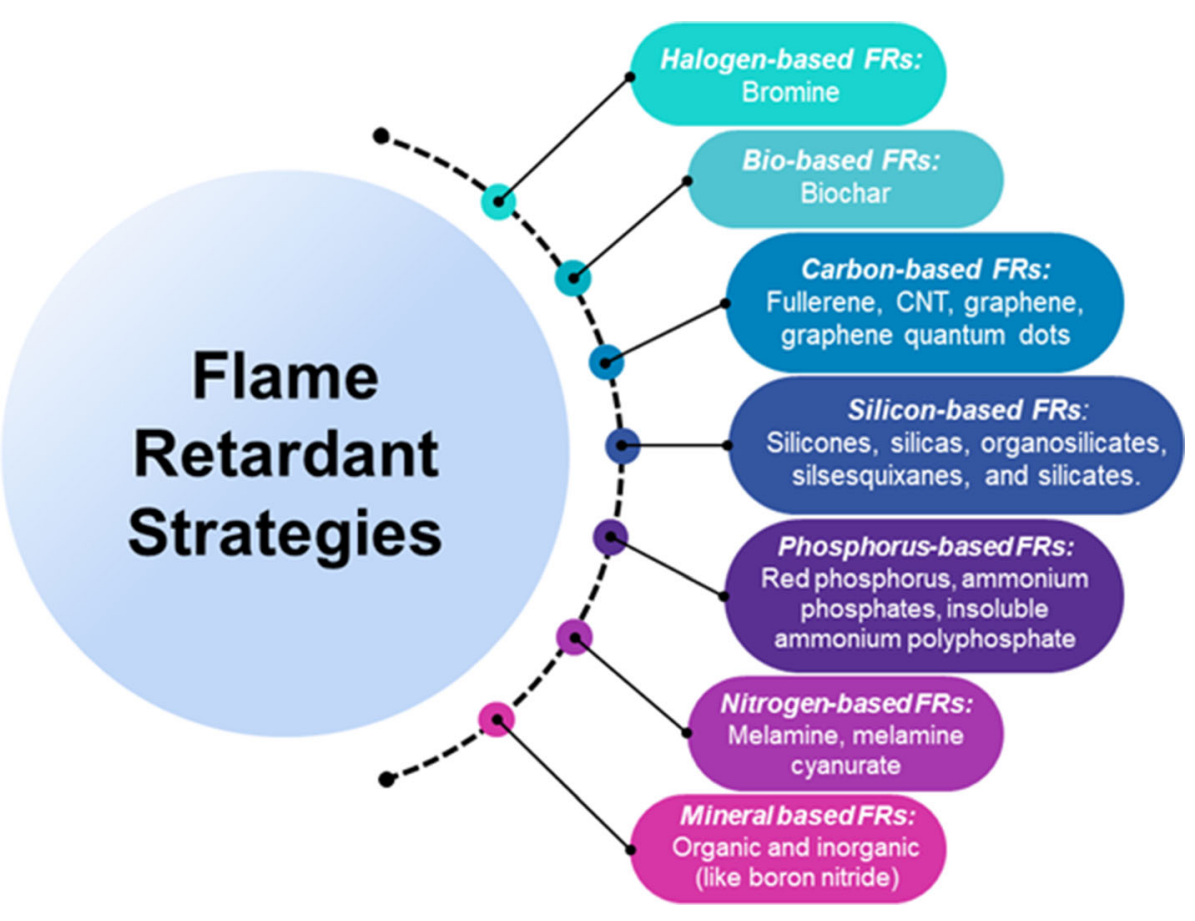

Fig. 2 Schematic figure showing different flame retardant strategies for polymeric materials 
in precise concentration is essential to exhibit balanced flame retardancy and mechanical strength of the 3D-printed parts.

Guo et al. (Ref 49) analyzed the flame retardancy of PLA samples manufactured by melt blending and FDM. Composites were fabricated by varying the weight percentages of PLA, melamine polyphosphate (MPP) and Cloisite 30B (C-30B). The authors produced different combinations: binary phase $(72 \%$ PLA $+18 \%$ MPP and $83 \%$ PLA $+17 \%$ MPP) and ternary phase $(82 \%$ PLA $+17 \%$ MPP $+1 \%$ C-30B and $81 \%$ PLA + $17 \%$ MPP $+2 \%$ C-30B) PLA samples using melt blending technique and studied their flammability properties using UL94 vertical burning test, limiting oxygen index (LOI) and cone calorimeter. In UL-94 and LOI tests, the neat PLA showed failrating (denoted as "NG") and 20\% LOI (vol.\%), respectively, thereby showing its high flammability. On the other hand, the $82 \%$ PLA $+17 \%$ MPP $+1 \%$ C-30B sample showed V-0 rating in UL94 test and displayed ca. 29\% LOI. In addition, the $82 \%$ PLA $+17 \%$ MPP $+1 \%$ C-30B sample made through melt blending technique displayed an average heat release rate (AHRR), PHRR and THR of $198,285 \mathrm{~kW} / \mathrm{m}^{2}$ and $84 \mathrm{MJ} / \mathrm{m}^{2}$, respectively, during cone calorimetry test and these were $2 \%$ (lower), $1 \%$ (higher) and 1\% (lower) than the similar PLA sample printed through FDM, respectively. It is interesting to note that the difference in manufacturing process did not influence the fire behavior of polymers.

The images of transmission electron microscopy (TEM) of the sample without cloisite displayed spherical shape of MPP, where the high interfacial energy restricted the deformation during the melt blending followed by the extrusion processes. Addition of 1 wt.\% cloisite, decreased the spherical size, however, the structure remained the same. Additionally, the addition of 2 wt.\% cloisite led to an enhancement of interfacial width reducing the interfacial energy. This resulted in the formation of softer MPP domains in the sample and made the blend more responsive to applied pressure (i.e., easily deformed) during the extrusion. Therefore, it is clear that the addition of $1 \mathrm{wt} \%$ cloisite is effective in reducing the MPP domains and achieving better dispersion but the addition of 2 wt.\% cloisite has shown an adverse effect, where the domains have grown larger and the degree of dispersion was reduced. This is one factor that partially explains the deterioration in flame retardant performance and the cone calorimetry response when the cloisite amount was increased from 1 to $2 \mathrm{wt} \%$ (see
Table 1). Another factor responsible for the poor performance of the 2 wt. $\%$ of cloisite added PLA was the aggregate formation inside the MPP domains, which partially blocked the contact between polyphosphate and the PLA chains. In the condensed phase, the polyphosphate component of MPP was able to catalyze the dehydration reaction of the polymer chains, which resulted in the formation of carbo-cations and $\mathrm{C}=\mathrm{C}$ bonds leading to the char formation. The excess C-30B platelets that remained aggregated within MPP domains hindered the catalysis reaction and the char formation was ineffective. In addition, it was found that the addition of MPP was essential for the formation of char, and only the chars corresponding to PLA $83 \%+$ MPP $17 \%$ and PLA $82 \%+$ MPP $17 \%+\mathrm{C}-30 \mathrm{~B} 1 \%$ were intumescent. The char corresponding to $\mathrm{P} 17 \mathrm{M} 2 \mathrm{C}$ was loose and powdery with no mechanical integrity. The authors also prepared PLA $82 \%+$ MPP 17\% + C-30B 1\% samples using Makerbot Replicator 2X - FDM 3D printer and compared the sample's performance with the earlier measures. It was noted that the 3D-printed PLA displayed V-0 rating during UL-94 test (Ref 49). The authors produced a complete report on flame retardancy mechanism and compared the flame retardancy of the $3 \mathrm{D}$-printed sample with conventionally extruded sample. The difference in HRR for the conventional sample and 3D-printed sample is provided in Fig. 3 as a guide to carry out further research on flame retardancy of 3D-printed samples with different flame retardant materials. A summary of the results from the above-mentioned research is presented in Table 1 .

Kuzman et al. (Ref 50) fabricated wood (unspecified species) reinforced PLA composites and analyzed their fire behavior. The authors planned to combine the advantages of 3D printing and natural resources and hence they utilised low cost and biodegradable materials wood (30 wt.\%) and PLA (70 wt.\%) as the constituents. PLA composites were printed with two different types of PLA filaments having two different wood reinforcements (light brown wood and dark brown wood) at 30 wt.\%. For the applied incident heat flux of $35 \mathrm{~kW} / \mathrm{m}^{2}$ (the authors used several incident heat fluxes), the PHRR of neat PLA, light brown wood/PLA and dark brown wood/PLA composites were ca. 404,319 and $246 \mathrm{~kW} / \mathrm{m}^{2}$, respectively. At the same incident heat flux, the dark brown wood/PLA displayed shorter TTI of $65 \mathrm{sec}$. It was followed by light brown wood/PLA composite (146 sec) and neat PLA (272 sec).

Table 1 Comparison of flame retardant polymers made by $3 D$ printing and conventional manufacturing

\begin{tabular}{|c|c|c|c|c|c|c|c|c|}
\hline Matrix & Reinforcement & $\begin{array}{c}\text { Technique of } \\
\text { manufacturing }\end{array}$ & $\begin{array}{c}\text { LOI, } \\
\%\end{array}$ & $\begin{array}{l}\text { TTI, } \\
\quad \mathbf{s}\end{array}$ & $\underset{\mathbf{m}^{2}}{\operatorname{AHRR}, \mathbf{k W} /}$ & $\begin{array}{c}\text { PHRR, kW/ } \\
\mathbf{m}^{2}\end{array}$ & $\begin{array}{c}\text { THR, MJ/ } \\
\text { m }^{2}\end{array}$ & Ref. \\
\hline \multirow[t]{4}{*}{ PLA } & None & Molding & 20 & 33 & 342 & 693 & 96 & Ref 49 \\
\hline & & $3 \mathrm{D}$ printing & 20 & 28 & 336 & 688 & 94.8 & \\
\hline & MPP (17 wt.\%) and C-30B (1 wt.\%) & Molding & 28.5 & 27 & 198 & 285 & 84 & \\
\hline & & $3 \mathrm{D}$ printing & 28.5 & 23 & 195 & 287 & 83.3 & \\
\hline \multirow[t]{6}{*}{ EVA } & None & Thermo-compression & $\ldots$ & 40 & $\ldots$ & 519 & 76 & Ref 56 \\
\hline & & $3 \mathrm{D}$ printing & $\ldots$ & 35 & $\ldots$ & 482 & 78 & \\
\hline & 65 wt. $\%$ ATH & Thermo-compression & $\ldots$ & 65 & $\ldots$ & 113 & 39 & \\
\hline & & 3D printing & $\ldots$ & 58 & $\ldots$ & 101 & 42 & \\
\hline & 10 wt. $\%$ EG & Thermo-compression & $\ldots$ & 21 & $\ldots$ & 156 & 63 & \\
\hline & & 3D printing & $\ldots$ & 18 & $\ldots$ & 217 & 75 & \\
\hline
\end{tabular}

LOI Limiting Oxygen Index; TTI Time-to-ignition; AHRR Average Heat Release Rate; PHRR Peak Heat Release Rate; THR Total Hear Release; PLA Poly (lactic acid), EVA Ethylene-vinyl acetate, MPP Melamine polyphosphate, ATH Aluminum trihydroxyde, EG Expandable graphite 


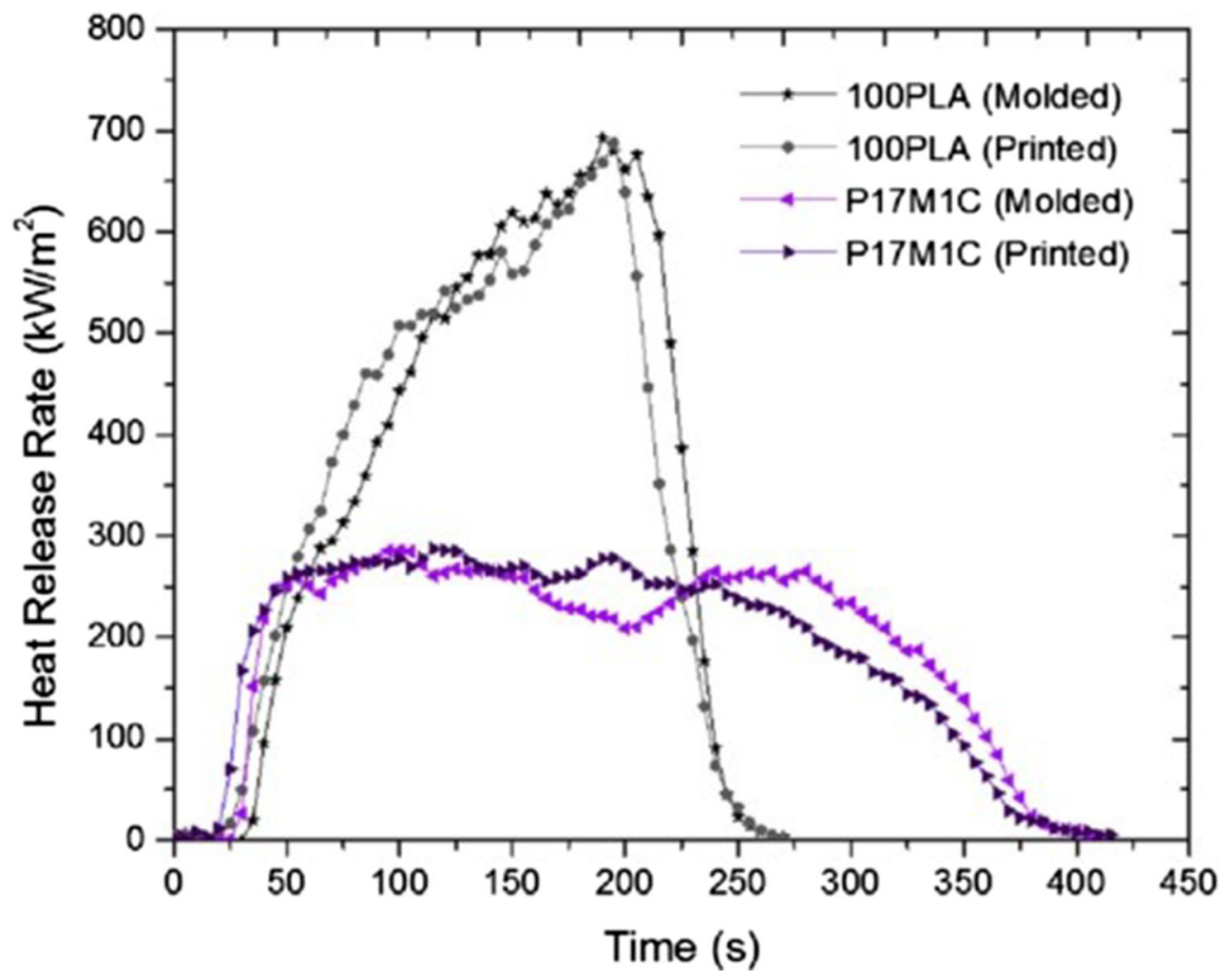

Fig. 3 HRRs of molded and 3D-printed samples as a function of heating time. Reprinted from Ref 49, with permission from Elsevier

This was because the wood (and other natural fibers) itself has very short TTI (Ref 51). It is to be kept in mind that for composites a longer TTI is desirable, which indicates that the material will take longer time to ignite, thereby, providing some form of safety from fire hazards. However, TTI is not the only parameter to judge the fire behavior of composite materials and other factors, such as PHRR, should also be considered. In this study, the authors observed that the dark brown wood/PLA composite had the lowest PHRR (at $35 \mathrm{~kW} / \mathrm{m}^{2}$ ) whereas the light brown wood/PLA composite had considerably higher PHRR. Although not mentioned by the authors, the most probable reason for the dark brown wood/PLA sample to have the lowest PHRR is due to a dense char formation, which can be seen from the two distinct HRR peaks as opposed to a shoulder and overlapped peak in the light brown wood/PLA samples. This means that the dark brown wood containing sample formed a more rigid char than the light brown counterpart. It is envisaged that the incorporation of FRs with wood/PLA composites would further lower the PHRR, making the composites more fire resistant.

\subsection{Ethylene-Vinyl Acetate (EVA)}

EVA is a non-degradable, biocompatible, non-toxic and insoluble elastomer. EVA is a copolymer of ethylene and vinyl acetate (VA), which has been approved by the Food and Drug Administration (FDA) to be used as a biomaterial (Ref 52). The amount of VA in an EVA copolymer can range between 0 and $40 \%$. The properties of the resultant EVA copolymer are dependent on the proportion of VA. High VA concentration leads to an increased polarity, adhesion, impact resistance, flexibility and compatibility of EVA with other polymers. At the same time, high VA concentration results in decreased crys- tallinity, stiffness and melting point of the copolymer (Ref 53). Filament buckling is the main problem associated with the processing of elastomers and this limits its application in FDM process. This buckling effect due to high flexibility making it difficult to process and extrude the elastomer via a small nozzle (Ref 54). However, various design implementations and modifications are proposed to print these flexible materials (Ref 55).

Geoffroy et al. (Ref 56) fabricated two different composites, namely, EVA copolymer with aluminum trihydroxyde (ATH) and EVA copolymer with expandable graphite (EG) using thermo-compression and $3 \mathrm{D}$ printing techniques and compared their flame retardancy properties. Mass loss calorimeter (MLC) was used to test the flame retardancy of the prepared EVA/ATH and EVA/EG samples. The neat EVA showed TTIs of 40s and $35 \mathrm{~s}$ for thermo-compressed and 3D-printed samples, respectively. The THR and PHRR for thermo-compressed neat EVA were $76 \mathrm{MJ} / \mathrm{m}^{2}$ and $519 \mathrm{~kW} / \mathrm{m}^{2}$, respectively; whereas for 3Dprinted neat EVA these were $78 \mathrm{MJ} / \mathrm{m}^{2}$ and $482 \mathrm{~kW} / \mathrm{m}^{2}$, respectively. The THR and PHRR were reduced by $49 \%$ and $78 \%$, respectively, when 65 wt. $\%$ of ATH was added in EVA and TTI was increased by $72 \%$ compared to neat EVA. The 65 wt.\% ATH added EVA composite showed better flameretardant performance than the 30 wt.\% ATH added EVA composite. This was majorly due to an endothermal dehydration that took place upon heating, resulting in the formation of the aluminum oxide $\left(\mathrm{Al}_{2} \mathrm{O}_{3}\right)$ ceramic residue. A critical amount of ATH is desirable to generate an effective homogenous residue, which acts as a fire barrier layer. This barrier formation was not accomplished at low ATH concentration (i.e., 30 wt.\%) (Ref 56). The research (Ref 56) reported the flame retardancy of two different composites but the mechanical properties were not revealed by the authors. In addition, the research (Ref 52) 
dealt with a single combination of 3D-printed polymer composites. In order to obtain balanced mechanical strength and flame retardancy, further optimisation studies have to be carried out.

A design modification was demonstrated by Geoffroy et al. (Ref 57) compared to the previously discussed literature (Ref 56) to accomplish light-weight 3D-printed parts with better flame retardancy. The design contains a core part, which was covered with an outer shell part. Firstly, the authors fabricated two different grid-patterned cores by varying infill density of either 30 or $50 \mathrm{wt} . \%$ and demonstrated the performance of two flame retardants (i.e., ATH and EG) used in the EVA. Secondly, the effect of biphasic materials containing air, water, solubilized or powdered potassium carbonate $\left(\mathrm{K}_{2} \mathrm{CO}_{3}\right)$, sodium carbonate $\left(\mathrm{Na}_{2} \mathrm{CO}_{3}\right)$ on the flame retardancy of $30 \mathrm{wt} . \%$ of ATH added EVA materials were evaluated. It was observed that 65 wt.\% ATH/EVA and 10 wt.\% EG/EVA materials displayed better flame retardancy than the $30 \mathrm{wt} . \%$ ATH/EVA, irrespective of the design studied. The PHRR of standard design plate (containing 100\% polymers) consisting of $30 \mathrm{wt} \%$ ATH/EVA was $88 \%$ and $150 \%$ higher than that of materials with the standard design containing 10 wt.\% EG/EVA and 65 wt.\% ATH/EVA, respectively. Furthermore, a remarkable improvement of flame retardancy was noticed in sandwich designs containing voids compared to $100 \%$ filled standard design plates irrespective of the material used. This was due to the presence of lower polymeric material in sandwich design compared to the standard design plates and consequently less fuel available for combustion. On one hand, there was no significant improvement in THR and PHRR when voids in the sandwich design were filled with $\mathrm{H}_{2} \mathrm{O}$ or powdered $\mathrm{K}_{2} \mathrm{CO}_{3}$ compared to $30 \mathrm{wt} . \%$ ATH/EVA material, which was filled with $70 \%$ air. Therefore, no benefit was achieved when air was replaced by $\mathrm{H}_{2} \mathrm{O}$ or powdered $\mathrm{K}_{2} \mathrm{CO}_{3}$. Instead, a significant decrease of PHRR (by $80 \%$ and $72 \%$ ), THR (by $75 \%$ and $71 \%$ ), and increase in TTI (by $31 \%$ and $42 \%$ ) were displayed when solutions with a same mass concentration of $\mathrm{K}_{2} \mathrm{CO}_{3}$ or $\mathrm{Na}_{2} \mathrm{CO}_{3}$ were used. The material with $\mathrm{K}_{2} \mathrm{CO}_{3}$ in liquid phase revealed the fastest flame extinguishment (due to $\mathrm{H}_{2} \mathrm{O}$ and $\mathrm{CO}_{2}$ emission and the release of $\mathrm{K}$ and $\mathrm{KOH}$ into the flame) and hence, this material showed the lowest HRR and THR when exposed to heat $(\operatorname{Ref} 57)$.
In the previous design, leakage of $\mathrm{H}_{2} \mathrm{O}$-based flame retardant was the major issue due to porosity of the polymeric matrix. To address this issue, Geoffroy et al. (Ref 58) introduced a novel multi-material sandwich incorporated with three different liquid hydrogels mixed with vermiculite (VMT) and vermiculite $/ \mathrm{K}_{2} \mathrm{CO}_{3}$ and assessed their flame-retardant performance using mass loss cone calorimeter test. The material was composed of (as shown in Figure 4) EVA/ATH (the squareshaped hollow cells at the middle, which is covered with two skin plates made of same polymer) and the hollow space in the cell zone filled with commercial fire extinguishing additive of saturated solution $\left(\mathrm{K}_{2} \mathrm{CO}_{3}\right)$. The incorporation of alginate hydrogel (HA) and HA+VMT both displayed longer TTI (6.5 and 7.5 times higher) as compared to air-filled 3D part. However, there was no significant improvement of THR and PHRR as compared to air-filled 3D part. For the 3D-printed multi-materials sandwich containing $\mathrm{HA}+\mathrm{VMT}+\mathrm{K}_{2} \mathrm{CO}_{3}$, the addition of $\mathrm{K}_{2} \mathrm{CO}_{3}$ led to a drastic reduction of THR and HRR. The THR of $\mathrm{HA}+\mathrm{VMT}+\mathrm{K}_{2} \mathrm{CO}_{3}$ system was reduced by $88 \%$, $66 \%$ and $88 \%$, compared to the air-filled reference part, $\mathrm{K}_{2} \mathrm{CO}_{3}$ saturated liquid-filled 3D part and 3D sample filled with $\mathrm{HA}+\mathrm{VMT}$, respectively. In addition, a decrease in PHRR by $86 \%, 38 \%$ and $84 \%$ was also observed for sample with $\mathrm{HA}+\mathrm{VMT}+\mathrm{K}_{2} \mathrm{CO}_{3}$ compared to air-filled 3D-printed part, and the sample filled with $\mathrm{K}_{2} \mathrm{CO}_{3}$ in liquid phase and $\mathrm{HA}+\mathrm{VMT}$, respectively. However, the sample containing a saturated $\mathrm{K}_{2} \mathrm{CO}_{3}$ solution showed 1.9 and 1.5 times higher TTI than the sample with air and $\mathrm{K}_{2} \mathrm{CO}_{3}$ saturated liquid. However, when compared to the sample HA + VMT the TTI was 3.9 times lower. The physical barrier formed by VMT platelets during the fire test, as well as the condensed phase mechanism of $\mathrm{K}_{2} \mathrm{CO}_{3}$ were found to be responsible for the excellent flame retardancy, as determined by confocal microscopy observations, electron probe micro analysis and x-ray diffraction experiments (Ref 58). This study developed routes for potential future research as there are opportunities to develop new 3Dprinted composites using alternate flame retardants such as graphene quantum dots, graphene and carbon nanotubes.

\subsection{Polyetherimide (PEI)}

PEI belongs to the special engineering thermoplastics with very good strength and thermal stability. PEI is an amorphous
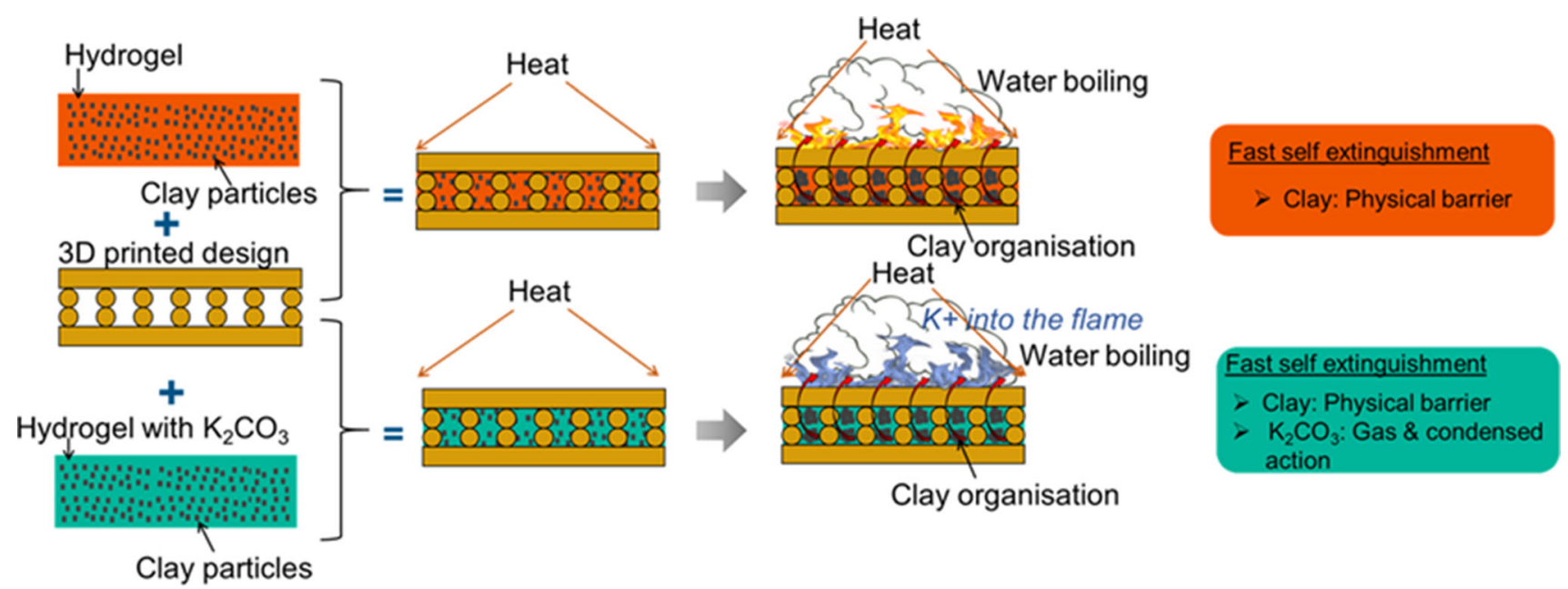

Fig. 4 Demonstration of sandwich technique for fast self-extinguishing 3D-printed part. Figure drawn on the basis of Ref 58 
polymer, which has primarily been used to manufacture highperformance electronic parts and biomaterials (Ref 59). Production of new materials with thermal stability has good potential (Ref 60) and is highly sought after for the design of thermal protection systems (TPS) used on space vehicles. The performance of these vehicles depends on TPS during hypersonic flight to guard against aerodynamic heating in atmospheric re-entry. Currently, the manufacturing of TPS is labor intensive and overall production costs can be high. However, the aerospace industry (both commercial and military areas) is already utilizing the benefits of $3 \mathrm{D}$ printing. For aerospace applications, 3D printing can significantly lower cost and production time when compared to conventional polymeric material processing methods (Ref 61). Developing materials that can be used to $3 \mathrm{D}$ print the heat shields for TPS will save both time and costs (Ref 62).

In this light, Wu et al. (Ref 63) developed novel hightemperature polymer composite filaments through extrusion process for application in FDM. Filaments were developed by altering PEI (ULTEM ${ }^{\mathrm{TM}} 1010$ resin) with the incorporation of functional fillers, namely hollow glass microspheres (HGMS), nanoclay (NC), and non-halogenated flame-retardant fillers. Three different filaments were extruded at varying proportions of fillers (PEI + 10 wt. $\%$ HGMS, PEI + 10 wt. $\%$ HGMS + 5 wt. $\%$ NC, PEI + 10 wt. $\%$ HGMS + 5 wt. $\%$ NC, 10 wt. $\%$ flame retardant) and their flame retardant properties were compared with neat PEI. The flame retardant (OP 1312) was a nonhalogenated additive composed of organic aluminum phosphinates. After burning, the PEI filament consisting $10 \mathrm{wt} \%$ HGMS, 5 wt.\% NC, and 10 wt.\% flame retardant fillers produced higher char yield of $62 \%$ than other filaments, which was $11 \%$ higher char than the neat PEI filament and exhibited low heat release capacity (HRC) of $119 \mathrm{~J} \mathrm{~g}^{-1} \mathrm{~K}^{-1}$ (52\% lower HRC than the neat PEI). In addition, the same filament composite showed 52\% reduced PHRR (W/g) and $30 \%$ reduced THR $(\mathrm{W} / \mathrm{g})$ than the neat $\mathrm{PEI}$ during microscale combustion calorimetry (MCC) test. The majority of the HGMS broke during combustion and the broken pieces acted as reinforcement to the already solid char layer, resulting in even lower flammability. The synergistic benefit of two manufacturing processes (extrusion and $3 \mathrm{D}$ printing) was simultaneously utilised in this study to demonstrate the performance of TPS. The results presented in this preliminary study will direct future optimisation and characterizations of FDM printed parts, which will provide a better understanding of the performance of PEI composite materials (Ref 63). For this purpose, composites with different fillers and filler effect on various polymers like PLA, EVA need to be investigated. Moreover, there are more opportunity to study flame retardancy of recycled 3D-printed polymer parts that will lead to increased reuse of waste polymers and cause reduced environmental pollution (Ref 64).

\subsection{Polyamide}

Polyamide- 6 is one of the major polymeric material used in engineering applications due to its good fiber-forming ability, biodegradability and biocompatibility (Ref 65). 3D-printed and electrospun polyamide polymers are potentially used in tissue engineering application due to its biocompatibility (Ref 66, 67). The printability of the material is highly dependent on the melt flow index (MFI), which conveys a measure of the ease of thermoplastic melts. MFI quantifies the weight of a thermo- plastic (in grams) melt in 10 min via a die of specific diameter and length with the application of pressure at a given temperature (Ref 68). Materials with an adequately high MFI (i.e., $>10 \mathrm{~g} / 10 \mathrm{~min}$ ) display good flow properties with no clogging at the nozzle (Ref 69) that lead to good finished printed part. Therefore, measurement of MFI of the polymer before and after the addition of flame retardant is highly informative to estimate its printability.

With this prospect, Wu et al. (Ref 70) fabricated flameretardant polyamide-6 (PA6) nanocomposites through FDM process. The MFI of both neat PA6 and the $15 \mathrm{wt} \% \%$ of flame retardant added PA6 nanocomposites was tested as per ASTM D1238. A drastic reduction in MFI was observed on the addition of flame retardant to the PA6 polymer. The neat PA6 showed an MFI value of $21.5 \mathrm{~g} / 10 \mathrm{~min}$, which was significantly higher than the MFI of flame retardant added PA6 (6.8 g/10 $\mathrm{min})$. This was due to the increased viscosity of the flame retardant PA6 compound, developed as a result of reduced chain movement induced by the formation of a nano-filler network. However, the increased melt viscosity of the nanocomposite sample did not significantly affect the filament processability, which means the flame retardant added PA6 nanocomposite was still found to be suitable for FDM printing. In addition, the thermal combustion properties of the printed PA6 and PA6 composite were tested according to ASTM D7309-2007 using the combustion calorimeter. The test was conducted at the combustion temperature of $900{ }^{\circ} \mathrm{C}$ and the heating rate of the pyrolysis was $1{ }^{\circ} \mathrm{C} / \mathrm{s}$. The heat release capacity and total heat release of the composite (ca. $900 \mathrm{~J} / \mathrm{g}-\mathrm{K}$ and ca. $60 \mathrm{~kJ} / \mathrm{g}$ ) was lower than the neat PA6 (ca.1300 J/g-K and ca. $63 \mathrm{~kJ} / \mathrm{g}$ ), which demonstrated the reduced flammability of the PA6 flame retardant composite.

\subsection{Silica Aerogels}

Silica aerogel is a porous and transparent solid material (Ref 71). Machining of silica aerogels is a challenging task due to its low density and high brittleness (Ref 72). Therefore, 3D printing of silica aerogels is a highly useful method to widen its applications. Maleki et al. (Ref 73) synthesized silk fibroin (SF) biopolymer, extracted from silkworm cocoons and added it to neat silica aerogels. In order to form stable covalent bonds between silica and SF structure at a molecular level, a carboxylic acid functional group-containing silane coupling agent as co-precursor was incorporated. The prepared hybrid silica-SF aerogel displayed an admirable printability in the wet state using a micro-extrusion-based 3D printing method. A significant enhancement in the mechanical strength (three-fold increase in stiffness) compared to the neat silica aerogel was achieved with minimal or almost zero compromise on the density and thermal insulation performance (Ref 73). Most importantly, the developed silica-SF aerogel had fire retardancy behavior and hence, did not require any additional flame retardant. Compared to halogen-based FR materials, silica aerogels are well-known fire-retardant materials that are considered to be innocuous toward human health (Ref 74). This innocuous nature of silica aerogel allows its utilization as a FR in composite preparation. In addition, hybrid form of silicaSF aerogel exhibited fire retardant behavior due to the homogeneous mixing of silica-SF network in the overall aerogel composite. The vertical burning experiment displayed some form of fire retardancy since the self-propagation of the flame did not happen and resulted in a carbonized residue with 
a retained shape and dimension as the original aerogel upon burning. However, the test for fire behavior was not conclusive since the authors did not report the rating of vertical burn test. In fact, it is not clear if they used proper standard for UL-94 test. Furthermore, the authors did not determine the LOI values as well as reaction-to-fire properties using cone calorimeter. The 3D-printed hybrid silica-SF aerogel composites have potential for a wide range of applications such as designing anisotropic thermal insulation materials. The simultaneous presence of micron and nano-sized pores provides an opportunity to develop scaffolds for bone-based regenerative medicine (Ref 73). However, it is important to study their flammability properties extensively to enhance their application in fire resistant applications.

\subsection{Contribution of Industries Toward Increasing Flame Retardancy of 3D-Printed Parts}

In this section available FR polymer filaments developed by industries are discussed. Research groups from well-known industrial 3D printer manufacturers are working to develop flame retardant reinforced plastics. For instance, the nylonbased carbon fiber filaments (trade name Onyx and Onyx FR) were developed by Markforged, Massachusetts, United States. The designed Onyx filament consisting of nylon mixed with chopped carbon fiber (Ref 75) showed tensile modulus, tensile stress at yield and tensile strain of $1.4 \mathrm{GPa}, 36 \mathrm{MPa}$ and $25 \%$, respectively. Onyx FR is plastic composite filament composed of flame retardant mixed continuous micro-carbon fiber and nylon. Compared to Onyx filament, the Onyx FR showed tensile modulus, tensile stress at yield and tensile strain of 1.3 GPa, $29 \mathrm{MPa}$ and 33\%, respectively. In addition, the Onyx FR filament obtained V-0 rating in UL-94 burning test down to a filament thickness of $3 \mathrm{~mm}$. The $\mathrm{V}-0$ rated flame retardant 3D printing plastic filament had good strength, high print quality with excellent surface finish and high resistance to fire. This combination of qualities makes Onyx FR fit for a wide range of applications in the aerospace clips and brackets, automotive, welding fixture, laser marking fixtures and defense industries, which often require non-flammable materials. The material was also designed to enable users to easily switch between different materials on the same $3 \mathrm{D}$ printer. This provides opportunity to examine the flame retardancy performance of various plastic composites (Ref 76). Recently, CRP technology, Modena, Italy introduced flame-retardant material known as Windform FR1 for additive manufacturing. It is carbon fiber reinforced flame retardant (UL 94 V-0 rated) material for additive manufacturing. The Windform FR1 underwent several tests such as Federal Aviation Regulations (FAR) 25.853 test, 12-second vertical and 15 -second horizontal flammability tests. The $45^{\circ}$ Bunsen burner test and the smoke density test have also been conducted that the material successfully passed. The Windform FR1 contains halogen-free polyamide-based material that combines good mechanical properties with lightweight nature. Owing to its flame retardancy performance, this high-performance polyamide-based composite material is suited for aircrafts and aerospace applications such as interior parts, cockpit, cabin components, air conditioning piping, air ducts, air outlet valves; automobile and transportation components such as vehicle interiors, housing and enclosure assemblies; consumer goods and electronics such as lighting, appliances and in general for any components demanding flame retardant standards (Ref 77).
The same company developed and introduced Windform FR2 composites, which contain polyamide-based material filled with halogen-free flame retardant and glass fiber and polyamide composites were fabricated by SLS process. Windform FR2 composites appear in white color and are electrically nonconductive. Windform FR2 combines high wear resistance and good temperature resistance. It allows for high detail resolution with a smoother surface finish than the Windform FR1. Similar to Windform FR1, this material also underwent series of flammability tests such as UL 94 horizontal burning of $1 \mathrm{~mm}$ and $3 \mathrm{~mm}$ rating, the FAR 25.853 12-second vertical and 15second horizontal flammability tests as well as the $45^{\circ}$ Bunsen burner test, smoke density test with successfully passing all of them (Ref 77, 78). These investigations by leading manufacturers of the $3 \mathrm{D}$ printers highlight the importance of the fire retardancy in $3 \mathrm{D}$ printing feedstock materials.

\section{Summary and Scope for Future Research}

This review article deals with the flammability characteristics of 3D-printed polymer and polymer composites. It is well known that 3D-printed composites have been studied in detail for their mechanical strength, and the available literature is also adequate in this area. Since, polymer and polymer composites are flammable, there is sufficient literature available to understand and address the flammability of polymers and their composites. However, there is dearth of studies on the fire behavior of 3D-printed polymers. This paves the way for the research community to develop flame retardant 3D-printed polymer composites and was the driving force behind this review article. From the review it is found that the available results are not enough to highlight the flammability behavior of 3D-printed materials. Following are some significant findings from the literature review:

- It should be noted that the variation in the printing parameter could have an effect on the flammability of 3Dprinted materials, which has not been investigated in detail. For example, critical FDM printing factors such as infill density, layer thickness and layer orientation, could alter the flammability of 3D-printed polymers and composites. However, some of the studies reported insignificant differences in flammability between $3 \mathrm{D}$ printing and conventional manufacturing process.

- Investigations on the flammability of 3D-printed polymer fiber composites are also inadequate. Most natural fiber reinforcements (except for wool) are expected to enhance the fire resistance of the polymer through an additive effect. Furthermore, the flammability properties of 3Dprinted materials could also vary due to the variation in fiber orientation and the reinforcement percentages, which need to be investigated in future research.

- The addition of a flame retardant could lead to a reduction in the strength of 3D-printed polymers, which should be studied in an interrelated approach between the flammability and the mechanical strength of the flame retardant added polymers and composites. Selection of a suitable matrix and fire retardant could warrant a balanced mechanical performance and flammability of the 3Dprinted polymer and its composites. 
- Apart from the flame retardant, the addition of nano fillers mainly carbon-based nano fillers such as graphene, carbon nanotubes, fullerene can provide effective mechanical strength and fire resistance, which should be studied in detail. Recent studies have examined the use of biochar reinforcement in polymers to increase fire resistance. Furthermore, biochar-based composites are sustainable and the development of biochar-based composites through FDM can simultaneously increase composite strength and fire retardancy with low production time and cost.

- Unlike conventional manufacturing methods, in 3D printing there is a chance for the development of pores and the loss of bonding of the layer, which lead to a reduction in the mechanical performance of the resulting components. The comparison of the flame retardant performance of the 3D-printed part with the conventionally produced should be performed comprehensively in future investigations.

- Most of the available literature did not report details such as PHRR, time to PHRR, THR, etc. It is therefore essential in the future, to report complete fire parameters, which would generate a holistic idea regarding the materials' flammability.

In conclusion, the available information for the aforementioned aspects are not sufficient to define, understand or presage the flammability of 3D-printed polymeric materials. Incentivising research into the issue will create mechanically sound and fire retardant 3D-printed parts and facilitate their foray into various engineering applications.

\section{Funding}

Open access funding provided by Lulea University of Technology.

\section{Open Access}

This article is licensed under a Creative Commons Attribution 4.0 International License, which permits use, sharing, adaptation, distribution and reproduction in any medium or format, as long as you give appropriate credit to the original author(s) and the source, provide a link to the Creative Commons licence, and indicate if changes were made. The images or other third party material in this article are included in the article's Creative Commons licence, unless indicated otherwise in a credit line to the material. If material is not included in the article's Creative Commons licence and your intended use is not permitted by statutory regulation or exceeds the permitted use, you will need to obtain permission directly from the copyright holder. To view a copy of this licence, visit http://creativecommons.org/licenses/by/4.0/.

\section{References}

1. N. Mohan, P. Senthil, S. Vinodh and N. Jayanth, A Review on Composite Materials and Process Parameters Optimisation for the Fused Deposition Modelling Process, Virtual Phys. Prototyp., 2017, 12, p 47-59

2. C.M. Choudhari and V.D. Patil, "Product Development and Its Comparative Analysis by SLA, SLS and FDM Rapid Prototyping Processes," IOP Conference Series: Materials Science and Engineering, Institute of Physics Publishing, 2016, p 012009
3. F.M. Mwema and E.T. Akinlabi, Basics of Fused Deposition Modelling (FDM), SpringerBriefs in Applied Sciences and Technology, Springer, 2020, p 1-15

4. T. Wohlers and T. Gornet, "History of Additive Manufacturing," 2016

5. M. Schmid, A. Amado, and K. Wegener, "Polymer Powders for Selective Laser Sintering (SLS)," AIP Conference Proceedings, American Institute of Physics Inc., 2015, p 160009

6. S. Liu, P. Zhao, S. Wu, C. Zhang, J. Fu and Z. Chen, A Pellet 3D Printer: Device Design and Process Parameters Optimization, $A d v$. Polym. Technol., 2019, 2019, p 1-8

7. V. Shanmugam, D.J. Johnson, K. Babu, S. Rajendran, A. Veerasimman, U. Marimuthu, S. Singh, O. Das, R.E. Neisiany, M.S. Hedenqvist, F. Berto and S. Ramakrishna, The Mechanical Testing and Performance Analysis of Polymer-Fibre Composites Prepared through the Additive Manufacturing, Polymer Test., 2020 https://doi.org/10.1016/j.polyme rtesting.2020.106925

8. V. Shanmugam, O. Das, K. Babu, U. Marimuthu, A. Veerasimman, D. Joel Johnson, R. Esmaeely Neisiany, M.S. Hedenqvist, S. Ramakrishna, and F. Berto, Fatigue Behaviour of FDM-3D Printed Polymers, Polymeric Composites and Architected Cellular Materials, International Journal of Fatigue.https://doi.org/10.1016/j.ijfatigue.2020.1060 07

9. J. Jing, Y. Zhang, X. Tang, Y. Zhou, X. Li, B.K. Kandola and Z. Fang, Layer by Layer Deposition of Polyethylenimine and Bio-Based Polyphosphate on Ammonium Polyphosphate: A Novel Hybrid for Simultaneously Improving the Flame Retardancy and Toughness of Polylactic Acid, Polymer, 2017, 108, p 361-371

10. T. Kashiwagi, F. Du, J.F. Douglas, K.I. Winey, R.H. Harris and J.R. Shields, Nanoparticle Networks Reduce the Flammability of Polymer Nanocomposites, Nat. Mater., 2005, 4(12), p 928-933

11. C.I. Idumah, A. Hassan and A.C. Affam, "A Review of Recent Developments in Flammability of Polymer Nanocomposites, Rev. Chem. Eng., 2015, 31, p 149-177

12. P. Wang, L. Xia, R. Jian, Y. Ai, X. Zheng, G. Chen and J. Wang, Flame-Retarding Epoxy Resin with an Efficient P/N/S-Containing Flame Retardant: Preparation, Thermal Stability, and Flame Retardance, Polym. Degrad. Stab., 2018, 149, p 69-77

13. M.F. Guigueno and K.J. Fernie, Birds and Flame Retardants: A Review of the Toxic Effects on Birds of Historical and Novel Flame Retardants, Environ. Res., 2017, 154, p 398-424

14. R.J. Jeng, S.M. Shau, J.J. Lin, W.C. Su and Y.S. Chiu, Flame Retardant Epoxy Polymers Based on All Phosphorus-Containing Components, Eur. Polym. J., 2002, 38(4), p 683-693

15. Q. Li, P. Jiang and P. Wei, Synthesis Characteristic, and Application of New Flame Retardant Containing Phosphorus, Nitrogen, and Silicon, Polym. Eng. Sci., 2006, 46(3), p 344-350

16. X. Qian, L. Song, Y. Bihe, B. Yu, Y. Shi, Y. Hu and R.K.K. Yuen, Organic/Inorganic Flame Retardants Containing Phosphorus Nitrogen and Silicon: Preparation and Their Performance on the Flame Retardancy of Epoxy Resins as a Novel Intumescent Flame Retardant System, Mater. Chem. Phys., 2014, 143(3), p 1243-1252

17. Y.Y. Yen, H.T. Wang and W.J. Guo, Synergistic Flame Retardant Effect of Metal Hydroxide and Nanoclay in EVA Composites, Polym. Degrad. Stab., 2012, 97(6), p 863-869

18. N. Hong, J. Zhan, X. Wang, A.A. Stec, T. RichardHull, H. Ge, W. Xing, L. Song and Y. Hu, Enhanced Mechanical, Thermal and Flame Retardant Properties by Combining Graphene Nanosheets and Metal Hydroxide Nanorods for Acrylonitrile-Butadiene- Styrene Copolymer Composite, Compos. Part A Appl. Sci. Manuf., 2014, 64, p 203-210

19. J. Chen, J. Wang, A. Ni, H. Chen, and P. Shen, Synthesis of a Novel Phosphorous-Nitrogen Based Charring Agent and Its Application in Flame-Retardant HDPE/IFR Composites, Polymers, 2019, 11(6), p 1062

20. X. Wang, E.N. Kalali, J.T. Wan and D.Y. Wang, Carbon-Family Materials for Flame Retardant Polymeric Materials, Prog. Polym. Sci., 2017, 69, p 22-46

21. M.M. Velencoso, A. Battig, J.C. Markwart, B. Schartel and F.R. Wurm, Molecular Firefighting-How Modern Phosphorus Chemistry Can Help Solve the Challenge of Flame Retardancy, Angew. Chem. Int. Ed., 2018, 57, p 10450-10467

22. M.S.S. Martins, B. Schartel, F.D. Magalhães and C.M.C. Pereira, The Effect of Traditional Flame Retardants, Nanoclays and Carbon Nanotubes in the Fire Performance of Epoxy Resin Composites, Fire Mater., 2017, 41(2), p 111-130 
23. M.J. Xu, S.Y. Xia, C. Liu, and B. Li, Preparation of Poly(Phosphoric Acid Piperazine) and Its Application as an Effective Flame Retardant for Epoxy Resin, Chin. J. Polym. Sci. 2018, 36(5), p 655-664

24. S. Huo, J. Wang, S. Yang, B. Zhang, X. Chen, Q. Wu and L. Yang, Synthesis of a Novel Reactive Flame Retardant Containing Phosphaphenanthrene and Piperidine Groups and Its Application in Epoxy Resin, Polym. Degrad. Stab., 2017, 146, p 250-259

25. O. Das, N.K. Kim, A.L. Kalamkarov, A.K. Sarmah and D. Bhattacharyya, Biochar to the Rescue: Balancing the Fire Performance and Mechanical Properties of Polypropylene Composites, Polym. Degrad. Stab., 2017, 144, p 485-496

26. O. Das, N.K. Kim, A.K. Sarmah and D. Bhattacharyya, Development of Waste Based Biochar/Wool Hybrid Biocomposites: Flammability Characteristics and Mechanical Properties, J. Clean. Prod., 2017, 144, p 79-89

27. E. Saleh, 3D and 4D Printed Polymer Composites for Electronic Applications, 3D and 4D Printing of Polymer Nanocomposite Materials: Processes, Applications, and Challenges, 2019, p 505-525

28. A.H. Espera, J.R.C. Dizon, Q. Chen, and R.C. Advincula, "3DPrinting and Advanced Manufacturing for Electronics," Progress in Additive Manufacturing, Springer, 2019, p 245-267

29. S.C. Joshi and A.A. Sheikh, 3D Printing in Aerospace and Its LongTerm Sustainability, Virtual Phys. Prototyp., 2015, 10(4), p 175-185

30. V. Babrauskas and R.D. Peacock, Heat Release Rate: The Single Most Important Variable in Fire Hazard, Fire Saf. J., 1992, 18(3), p 255-272

31. A.B. Morgan, The Future of Flame Retardant Polymers-Unmet Needs and Likely New Approaches, Polym. Rev., 2019, 59, p 25-54

32. S. Vigneshwaran, R. Sundarakannan, K.M. John, R.D. Joel Johnson, K.A. Prasath, S. Ajith, V. Arumugaprabu, and M. Uthayakumar, "Recent Advancement in the Natural Fiber Polymer Composites: A Comprehensive Review," J. Clean. Prod., 2020, p 124109, https://doi. org/10.1016/j.jclepro.2020.124109

33. R.E. Lyon and M.L. Janssens, Polymer Flammability, Encyclopedia of Polymer Science and Technology, Wiley, Hoboken, 2015, p 1-70

34. J. Gardan, Additive Manufacturing Technologies: State of the Art and Trends, Int. J. Prod. Res., 2016, 54(10), p 3118-3132

35. T.N.A.T. Rahim, A.M. Abdullah, and H. Md Akil, "Recent Developments in Fused Deposition Modeling-Based 3D Printing of Polymers and Their Composites," Polymer Reviews, Taylor and Francis Inc., 2019, p 589-624

36. B. Dittrich, K.-A. Wartig, R. Mülhaupt and B. Schartel, FlameRetardancy Properties of Intumescent Ammonium Poly(Phosphate) and Mineral Filler Magnesium Hydroxide in Combination with Graphene, Polymers, 2014, 6(11), p 2875-2895

37. M. Yurddaskal and E. Celik, Effect of Halogen-Free Nanoparticles on the Mechanical Structural, Thermal and Flame Retardant Properties of Polymer Matrix Composite, Compos. Struct., 2018, 183(1), p 381388

38. M. Rakotomalala, S. Wagner and M. Döring, Recent Developments in Halogen Free Flame Retardants for Epoxy Resins for Electrical and Electronic Applications, Materials, 2010, 3(8), p 4300-4327

39. B. Perret, B. Schartel, K. Stöß, M. Ciesielski, J. Diederichs, M. Döring, J. Krämer and V. Altstädt, Novel DOPO-Based Flame Retardants in High-Performance Carbon Fibre Epoxy Composites for Aviation, Eur. Polym. J., 2011, 47(5), p 1081-1089

40. Y. Chen and Q. Wang, Preparation, Properties and Characterizations of Halogen-Free Nitrogen-Phosphorous Flame-Retarded Glass Fiber Reinforced Polyamide 6 Composite, Polym. Degrad. Stab., 2006, 91(9), p 2003-2013

41. "Research Progress on Silicon-Based Flame Retardants-Chemical Industry and Engineering Progress, 2003年08期,” n.d

42. L. Costes, F. Laoutid, S. Brohez, and P. Dubois, "Bio-Based Flame Retardants: When Nature Meets Fire Protection," Materials Science and Engineering R: Reports, 2017, p 1-25

43. K. Babu, G. Rendén, R. Afriyie Mensah, N.K. Kim, L. Jiang, Q. Xu, Á. Restás, R. Esmaeely Neisiany, M.S. Hedenqvist, M. Försth, A. Byström, and O. Das, A Review on the Flammability Properties of Carbon-Based Polymeric Composites: State-of-the-Art and Future Trends, Polymers, 2020, 12(7), p 1518, https://doi.org/10.3390/poly m12071518

44. S.C. Ligon, R. Liska, J. Stampfl, M. Gurr, and R. Mülhaupt, "Polymers for 3D Printing and Customized Additive Manufacturing," Chem. Rev., 2017, p 10212-10290
45. B. Coppola, N. Cappetti, L. Di Maio, P. Scarfato, and L. Incarnato, 3D Printing of PLA/Clay Nanocomposites: Influence of Printing Temperature on Printed Samples Properties, Materials, 2018, 11(10), p 1947

46. D. Bai, H. Liu, H. Bai, Q. Zhang and Q. Fu, Powder Metallurgy Inspired Low-Temperature Fabrication of High-Performance Stereocomplexed Polylactide Products with Good Optical Transparency, Sci. Rep., 2016, 6(1), p 1-9

47. K.C. Cheng, C. Bin Yu, W. Guo, S.F. Wang, T.H. Chuang, and Y.H. Lin, Thermal Properties and Flammability of Polylactide Nanocomposites with Aluminum Trihydrate and Organoclay, Carbohydrate Polymers, 2012, 87(2), p 1119-1123

48. N. Najafi, M.C. Heuzey and P.J. Carreau, Polylactide (PLA)-Clay Nanocomposites Prepared by Melt Compounding in the Presence of a Chain Extender, Compos. Sci. Technol., 2012, 72(5), p 608-615

49. Y. Guo, C.C. Chang, M.A. Cuiffo, Y. Xue, X. Zuo, S. Pack, L. Zhang, S. He, E. Weil and M.H. Rafailovich, Engineering Flame Retardant Biodegradable Polymer Nanocomposites and Their Application in 3D Printing, Polym. Degrad. Stab., 2017, 137, p 205-215

50. M. K. Kuzman, M. Kariz, N. Ayrilmis, M. Šernek, J. Žigon, and Q. Xu. Fire Behaviour of 3D-printed PLA and Wood/PLA Composites. Digitalisation and Circular Economy, 2019, 149

51. O. Das, N.K. Kim, M.S. Hedenqvist, R.J.T. Lin, A.K. Sarmah and D. Bhattacharyya, An Attempt to Find a Suitable Biomass for BiocharBased Polypropylene Biocomposites, Environ. Manag., 2018, 62(2), p 403-413. https://doi.org/10.1007/s00267-018-1033-6

52. C. Schneider, R. Langer, D. Loveday, and D. Hair, "Applications of Ethylene Vinyl Acetate Copolymers (EVA) in Drug Delivery Systems," J. Controlled Release, 2017, p 284-295

53. N. Genina, J. Holländer, H. Jukarainen, E. Mäkilä, J. Salonen and N. Sandler, Ethylene Vinyl Acetate (EVA) as a New Drug Carrier for 3D Printed Medical Drug Delivery Devices, Eur. J. Pharm. Sci., 2016, 90, p 53-63

54. N. Kumar, P.K. Jain, P. Tandon, and M. Pandey, 3D Printing of Flexible Parts Using EVA Material, 2018

55. N. Kumar, P.K. Jain, P. Tandon and P.M. Pandey, The Effect of Process Parameters on Tensile Behavior of 3D Printed Flexible Parts of Ethylene Vinyl Acetate (EVA), J. Manuf. Process., 2018, 35, p 317326

56. L. Geoffroy, F. Samyn, M. Jimenez and S. Bourbigot, Additive Manufacturing of Fire-retardant Ethylene-vinyl Acetate, Polym. Adv. Technol., 2019, 30(7), p 1878-1890

57. L. Geoffroy, F. Samyn, M. Jimenez and S. Bourbigot, Innovative 3D Printed Design to Conceive Highly Fire-Retardant Multi-Material, Polym. Degrad. Stab., 2019, 169, p 108992

58. L. Geoffroy, A. Lise Davesne, S. Bellayer, F. Blanchard, E. Richard, F. Samyn, M. Jimenez, and S. Bourbigot, 3D Printed Sandwich Materials Filled with Hydrogels for Extremely Low Heat Release Rate, Polym. Degrad. Stab., 2020, 179, p 109269

59. S. Ding, B. Zou, P. Wang and H. Ding, Effects of Nozzle Temperature and Building Orientation on Mechanical Properties and Microstructure of PEEK and PEI Printed by 3D-FDM, Polym. Test., 2019, 78, p 105948

60. A. Veerasimman, V. Shanmugam, S. Rajendran, D.J. Johnson, A. Subbiah, J. Koilpichai, and U. Marimuthu, Thermal Properties of Natural Fiber Sisal Based Hybrid Composites-A Brief Review, J. Natl. Fibers, 2021, p 1-11, https://doi.org/10.1080/15440478.2020.18 70619

61. M.Q. Chu, L. Wang, H.Y. Ding and Z.G. Sun, Additive Manufacturing for Aerospace Application, Appl. Mech. Mater., 2015, 798, p 457-461

62. W.P. Fahy, J.H. Koo, S. Kim, A. Devega, M. Sico, H. Wu, W. Fahy, and K. Holder, Evaluation of a Modified Fused Filament Fabrication Material for Use As Thermal Protection, 2019

63. H. Wu, M. Sulkis, J. Driver, A. Saade-Castillo, A. Thompson, and J.H. Koo, Multi-Functional ULTEM $^{\mathrm{TM}} 1010$ Composite Filaments for Additive Manufacturing Using Fused Filament Fabrication (FFF), Addit. Manuf., 2018, 24, p 298-306

64. V. Shanmugam, O. Das, R.E. Neisiany, K. Babu, S. Singh, M.S. Hedenqvist, F. Berto, and S. Ramakrishna, Polymer Recycling in Additive Manufacturing: An Opportunity for the Circular Economy, Mater. Circ. Econ. 2020, 2(1), p 11, https://doi.org/10.1007/s42824020-00012-0

65. J. Matulevicius, L. Kliucininkas, D. Martuzevicius, E. Krugly, M. Tichonovas, and J. Baltrusaitis, Design and Characterization of 
Electrospun Polyamide Nanofiber Media for Air Filtration Applications, J. Nanomater., 2014, 2014

66. B.K. Shrestha, H.M. Mousa, A.P. Tiwari, S.W. Ko, C.H. Park and C.S Kim, Development of Polyamide-6,6/Chitosan Electrospun Hybrid Nanofibrous Scaffolds for Tissue Engineering Application, Carbohydr. Polym., 2016, 148, p 107-114

67. K.C. Hung, C.S. Tseng and S.H. Hsu, Synthesis and 3D Printing of Biodegradable Polyurethane Elastomer by a Water-Based Process for Cartilage Tissue Engineering Applications, Adv. Healthc. Mater., 2014, 3(10), p 1578-1587

68. A. Riley, Plastics Manufacturing Processes for Packaging Materials, Packaging Technology, Elsevier, Amsterdam, 2012, p 310-360

69. S. Wang, L. Capoen, D.R. D'hooge, and L. Cardon, Can the Melt Flow Index Be Used to Predict the Success of Fused Deposition Modelling of Commercial Poly(Lactic Acid) Filaments into 3D Printed Materials?, Plast. Rubber Compos., 2018, 47(1), p 9-16

70. H. Wu, A. Kafi, H. Kim, R. Shah, S. Bateman, J.H. Koo, and A.T. Usa, "Additive Manufacturing of Flame-Retardant Polyamide 6 Nanocomposites via Fused Filament Fabrication (FFF)," 2019

71. M. Cantin, M. Casse, L. Koch, R. Jouan, P. Mestreau, D. Roussel, F. Bonnin, J. Moutel and S.J. Teichner, Silica Aerogels Used as Cherenkov Radiators, Nucl. Instrum. Methods, 1974, 118(1), p 177182
72. S. Saeed, R.M. Al-Sobaihi, M.F. Bertino, L.S. White and K.M. Saoud, Laser Induced Instantaneous Gelation: Aerogels for 3D Printing, $J$. Mater. Chem., 2015, 3(34), p 17606-17611

73. H. Maleki, S. Montes, N. Hayati-Roodbari, F. Putz and N. Huesing, Compressible, Thermally Insulating, and Fire Retardant Aerogels through Self-Assembling Silk Fibroin Biopolymers Inside a Silica Structure-An Approach towards 3D Printing of Aerogels, ACS Appl. Mater. Interfaces, 2018, 10(26), p 22718-22730

74. T. Kashiwagi, J.W. Gilman, K.M. Butler, R.H. Harris, J.R. Shields and A. Asano, Flame Retardant Mechanism of Silica Gel/Silica, Fire Mater, 2000, 24(6), p 277-289

75. S.M.F. Kabir, K. Mathur, and A.-F.M. Seyam, The Road to Improved Fiber-Reinforced 3D Printing Technology, Technologies, 2020, 8(4), p 51

76. "Onyx FR - Flame Resistant 3D Printing Material." https://markforg ed.com/materials/plastics/onyx-fr

77. "CRP Technology Launches Flame-Retardant Windform FR1." http:// www.windform.com/windform-fr1.html

78. "Windform FR2. Composite Polyamide Based Material Glass Fiber Filled." http://www.windform.com/windform-fr2.html

Publisher's Note Springer Nature remains neutral with regard to jurisdictional claims in published maps and institutional affiliations. 